\title{
Dental Image Analysis Approach Integrates Dental Image Diagnosis
}

\section{IJCRR}

Section: Healthcare

Sci. Journal Impact

Factor: 6.1 (2018)

ICV: 90.90 (2018)

(c) (i) (5)

Copyright@IJCRR

\section{Mohd Abdul Khader Khan'1, M V Aditya Nag2 ${ }^{2}$ Tahir ul Gani Mir ${ }^{3}$, Sapna Dhiman}

'Professor, Department of ECE, Shadan Women's College of Engineering and Technology, Hyderabad, Telangana, India; ${ }^{A}$ Assistant Professor, Department of Mechanical Engineering, Institute of Aeronautical Engineering, Hyderabad, Telangana, India; ${ }^{3}$ Department of Forensic Sciences, Lovely professional University, Phagwara, Punjab, India; ${ }^{4}$ Assistant Professor, Department of Computer Science, Multani Mal Modi College, Patiala, Punjab, lndia.

\section{ABSTRACT}

Introduction: Dentistry is now a big human issue for a day and causes enormous teeth pain. Dental caries is also known as caries and tooth decay, and acids produced by bacteria can cause the loss of teeth. Cavities can be seen from yellow to black in various colours. Symptoms include discomfort without apparent cause, trouble feeding and clear trousers or holes in the teeth.

Method: Dental X-ray is useful for dentists, for example, root canal treatment, caries identification and many other abnormalities. The procedure is performed with the awareness of the tooth if it is affected by tooth decay. We will better handle the length of the infected tooth-root canal. Dentists had difficulties in previous years in identifying the teeth and the CNN(Computer Neural Network) technology helps us to accurately distinguish the extreme dental tooth and the root channel length from $\mathrm{x}$-rays.

Result: The pictures are pre-processed, segmented and then tooth parameters are removed. Deep learning is widely used as a teacher's tool where a neural network will learn features automatically. For picture analysis, evolutionary networks are widely used. This refers to areas like segmentation, identification of anomalies and diagnosis of diseases.

Conclusion: In addition to high heterogeneity, database with several images implemented. Due to this heterogeneity in the database with the number of images, the powerful methodology is required to increase the compatibility and robustness between the studies, neural networks architecture and its results. The proposed Neural network approach detected Dental problems with higher accuracy.

Key Words: Deep Learning, Medical Image Processing, Dental application, Detection

\section{INTRODUCTION}

In dentistry medical imaging techniques such an X-Ray or Computer Neural Network(CT) Scan are using from the past decades to detect the problems and identifying the treatment options for various diseases. To help the Dental Professionals in identifying and treating such diseases, computer programs plays a vital role nowadays. Man-made brainpower portrays calculations intended critical thinking. The accomplishment of deep learning for the most part because of the advancement in the Personal Computer(PC) limit, the tremendous measure of information accessible, and improvement of calculations. This technique has been demonstrated and is utilized adequately in a picture-based determination in a few fields. Convolutional neural network systems (CNNs) are generally utilized depending on deep realizing, grown very rapidly most recent, primarily decision breaking down clini- cal pictures. ${ }^{1}$ CNN's have been effectively utilized in medication, basically in malignancy, for the mechanized evaluation of bosom disease, skin malignant growth assessments.

Sorts of neural systems utilized recognize structures, for example, teeth or caries, to order them, and to portion them. ${ }^{2}$ Neural systems should be prepared and streamlined, and for that a picture database is essential. A few picture strategies in the dentistry field contingent upon utilization. Pictures utilized to catch unblemished teeth, including front and back, just as their encompassing bone; along these lines, periapical pictures are exceptionally useful to imagine expected caries, periodontal bone misfortune, and periapical ailments. All-encompassing radiographic are basic in dentistry since they take into consideration the screening of an expansive anatomical area and simultaneously, requires a generally low radiation portion. ${ }^{3}$ The goal

\section{Corresponding Author:}

Dr. M V Aditya Nag, Assistant Professor, Department of Mechanical Engineering, Institute of Aeronautical Engineering, Hyderabad, Telangana, India; Contact: +918464843058; Email: aditya.iare147@gmail.com

ISSN: 2231-2196 (Print) ISSN: $0975-5241$ (Online)

Received: 09.06.2020

Revised: 11.07 .2020

Accepted: 03.08.2020

Published: 22.08 .2020 
is to envision the best in a class of man-made brainpower in different dental applications.

\section{LITERATURE STUDY}

Deep learning techniques can be utilized as they can help in highlight determination, extraction and can likewise make new highlights. The Artificial Intelligence (AI) is characterized as an assortment of apparatuses that can distinguish designs in the information and afterwards utilize those examples for forecast and help in dynamic. ${ }^{4} \mathrm{AI}$ and man-made reasoning methods can be applied in different regions of the clinical field like clinical picture handling, clinical picture translation, picture division, picture combination, picture enrollment, picture recovery, picture guided treatment, and can extricate and speak to data from picture effectively, precisely and proficiently. These strategies help the specialists to analyze and foresee the danger of maladies quicker and precise. It encourages them to examine the nonexclusive changes than can prompt ailment. These methods include traditional neural system, intermittent neural system, long transient memory, Deep Neural Network, Deep Conventional, Extreme Learning Machine, Generative ill-disposed systems, Deep Boltzmann Machine, Deep Belief Network, Deep Auto Encoder, and so on. ${ }^{5}$

Deep learning can be characterized as a unique kind of fake neural system that looks to some extent like the human dynamic procedure. ${ }^{6}$ It is getting high consideration for its utilization in huge information in social insurance. The primary counterfeit neural system was presented in the mid-1950s but since of its impediments like overfitting issues, evaporating slope, and so forth slowed down the preparation of the engineering ${ }^{7}$. Furthermore around then, there was an absence of processing power and adequate information to give preparing to the framework.

\section{PROPOSED SYSTEM}

The proposal was to envision the best in the class of manmade consciousness in distinguishing different dental circumstances. Neural systems have single or various layers, ${ }^{8}$ with hubs or neurons, interconnected permits signs to go through the system. ANNs are normally partitioned into three layers of neurons, to be specific: input (gets the data), covered up (extricates designs and plays out the inner preparing), and yield (presents the last system yield). Preparing is the procedure to upgrade boundaries. ${ }^{9}$ The system architecture for teeth detection is shown in Figure 1. An everincreasing number of businesses are utilizing man-made brainpower to settle on progressively complex choices, and numerous choices are accessible to them. Be that as it may, taking into account our outcomes, there is a lack of direction on choosing the suitable techniques ${ }^{10}$ custom fitted to the social insurance industry.

Dental Implant and Filled Teeth Detection: Implant treatment is a typical practice in various clinical circumstances for supplanting teeth. ${ }^{11}$ In any case, no investigations discovered utilized man-made reasoning and neural systems to identify inserts on radiographs. The equivalent is valid for filled teeth discovery.

Endodontic Treatment Detection: A convolutional neural system $(\mathrm{CNN})$ framework ${ }^{12}$ utilized by Fukuda et al. for distinguishing vertical root fractures (VRFs) in all-encompassing radiographers. 300 pictures were utilized as a picture dataset, of which 240 pictures relegated to a preparation set and 60 pictures allowed to a test set. ${ }^{13}$ This examination built CNN-based deep learning model utilizing DetectNet with DIGITS form 5.0 (city and nation) and acquired review of 0.75 , a mean accuracy of 0.93 , and a proportion of 0.83 .

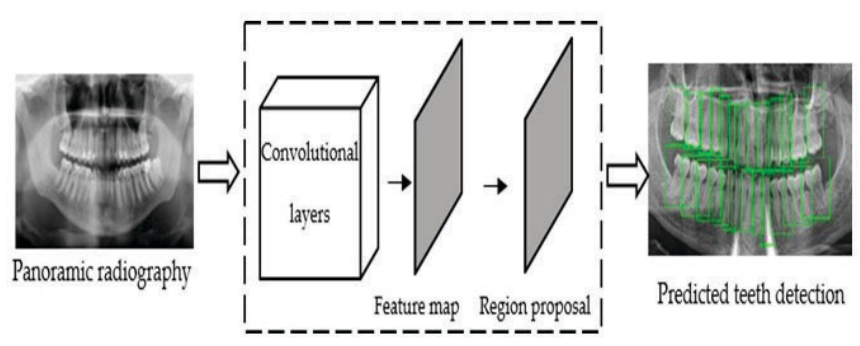

Figure 1: System architecture for teeth detection.

\section{METHODOLOGY}

The advantage of neural systems in medication and dentistry is identified with their capacity to process a lot of information for breaking down, diagnosing, and infection checking. Deep learning an incredible partner in the field of medication when all is said in done and is starting to be one in dentistry. The Automated analysis of dental images for clinical outcome evaluation is shown in figure 2 , the architecture shown in figure 1 replicates the main procedure following in this proposed research work and is a general exploration strategy examination of our study. ${ }^{14}$ The initial step is to get a trial dataset. The dental specialists gave a named dataset, which comprised of 196 sets of periapical radiography pictures taken when treatment. Our master group incorporates three expert dental specialists and a central radiologist. We extricate the ROIs - the apical area in picture preprocess technique: We first utilize the SIFT and SURF calculations to discover include focuses between the pair of pictures. At that point, we locate the best ternary coordinating focuses utilizing the base greyscale contrast strategy. From that point forward, we ascertain the relative grid dependent on the best ternary focuses and utilize the picture after the relative change to do 
picture deduction. We misuse channel filling districts, which is clear in pictures, assist us with finding the root locale. ${ }^{15}$

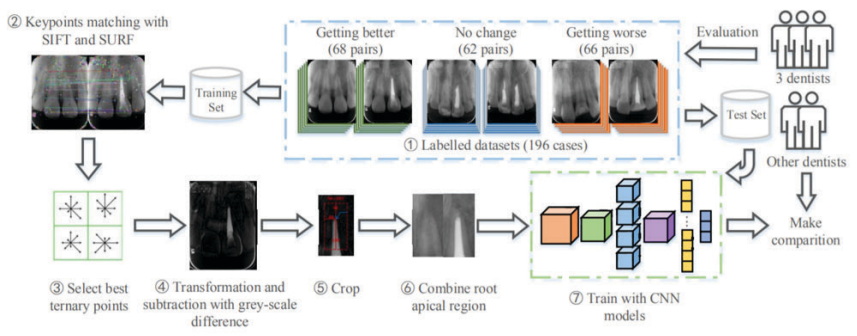

Figure 2: Automated analysis of dental images for clinical outcome evaluation.

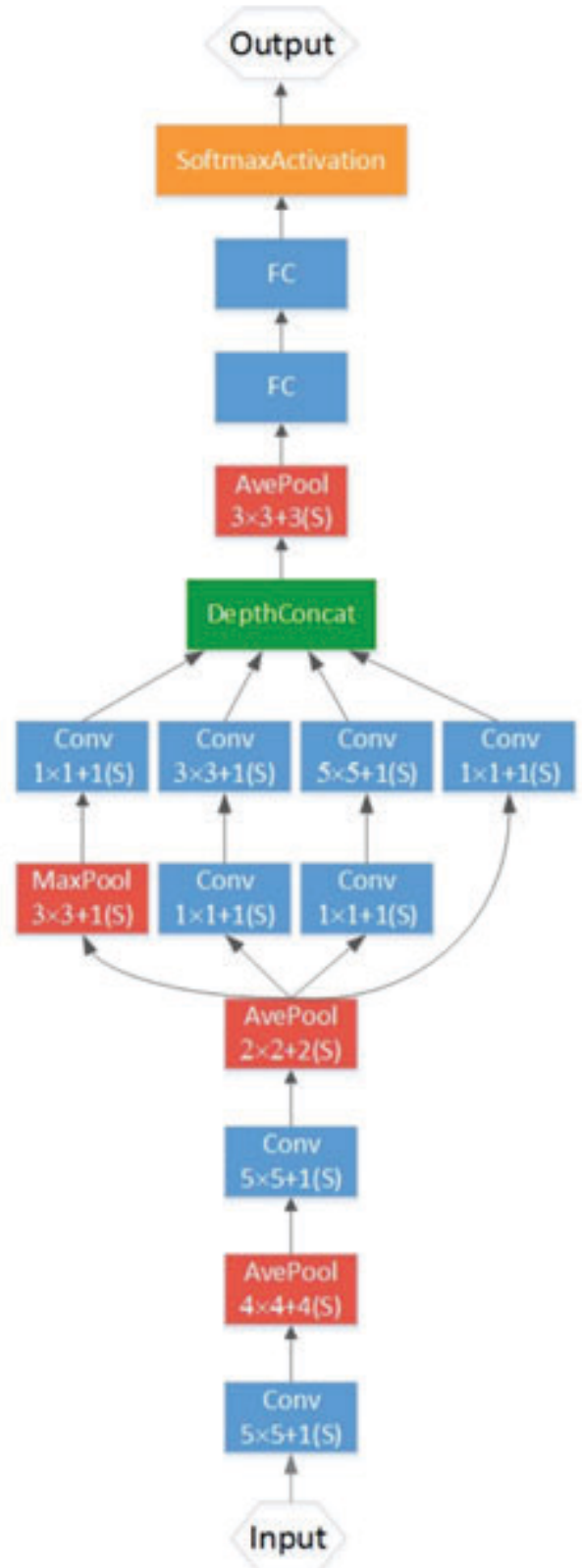

Figure 3: The architecture of the deep neural network.
The principle preferences of these kinds of pictures are the patient solace contrasted and different strategies, for example, intraoral pictures (bitewing and periapical); the low radiation introduction; capacity assess bigger zone of maxilla and mandible. ${ }^{16}$ All-encompassing radiographs are helpful to assess endodontic medicines, periapical injuries, and issues in bones, among others.

The architecture of the deep neural network shown in Figure 3 , is used for achieving the outcome of the currently proposed method. The cropped images are applied as input to this network after performing pre-processing. This architecture is made with a total of four Convolutional layers in which two are traditional, two full connected layers along with a softmax layer. The kind of picture has gotten the best outcomes in tooth identification on the off chance that we contrast it and the examination that utilized periapical pictures to identify this variable. What's more, the outcomes got by the investigations that recognized teeth were better than the remainder of the factors broke down, paying little heed to the system or sort of picture utilized. Caries is one of the most widely recognized constant maladies in the oral field, an extraordinary effect on a patient's wellbeing.

\section{EXPERIMENTAL RESULT}

Datasets: We recognized and commented on the dataset of 196 sets of periapical dental radiographs. Each radiograph contains at least one teeth for treatment and each pair comprises two radiographs taken pre-and post-treatment. Experienced dental specialists mark these teeth as 'showing signs of improvement', 'no change', or 'deteriorating' contingent upon clinical perception. During the assessment, radiologists may make slight alterations dependent on this setting. The shooting edge of every tooth is unique, known as the bisecting point procedure. To advance the dataset, we play out an information enlargement method. During the time spent radio-graphical assessment, the nature of pictures will be impacted by factors like introduction time, radiation point, and so on. We flip the picture on a level plane and vertically, make slow turns, and change the picture brilliance inside a specific range to mimic various conceivable assessment conditions in reality.

Automated Calibration and Cropping of Apical Region: In the underlying test, we use pictures to incorporate the whole tooth as info. Be that as it may, cases like contiguous tooth cover under X-beam and tooth wear acquaint clamour with the issue. Then again, to abstain from overfitting, we need to lessen the size of info pictures because of the constrained example cases in the dataset. As indicated by the dental specialists' understanding, we extricate the apical area ${ }^{17}$. To get the substracted dental 
image, we need to find out the matching points first by considering the input image as shown in Figure 4.

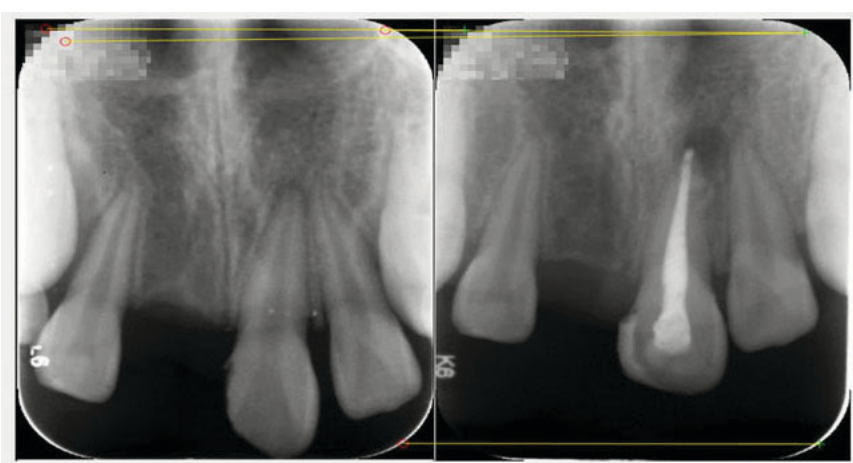

Figure 4: Input image.

The detection of candidate point matches is shown in Figure 5. These feature matching points are found using Scale Invariant Feature Transform (SIFT) and Speed Up Robust Features (SURF) which are indicated in Figure 2.

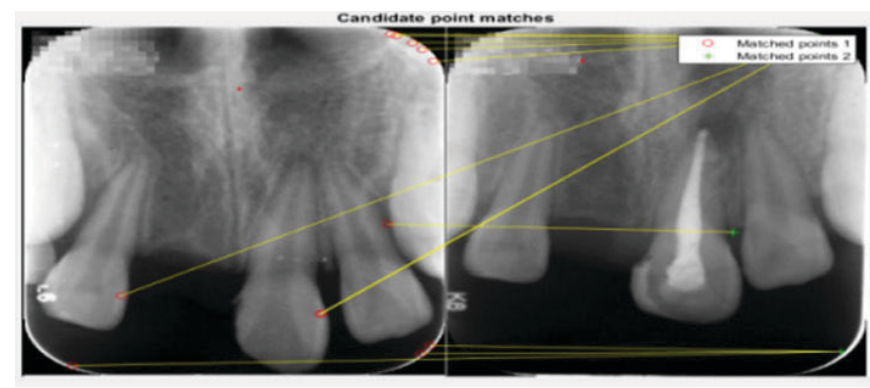

Figure 5: Detection of Point matches.

The cropping of an image apical portion is done by the proposed architecture ${ }^{18}$. The proposed architecture has greater accuracy and covers 12000 iterations as shown in figure 6 .

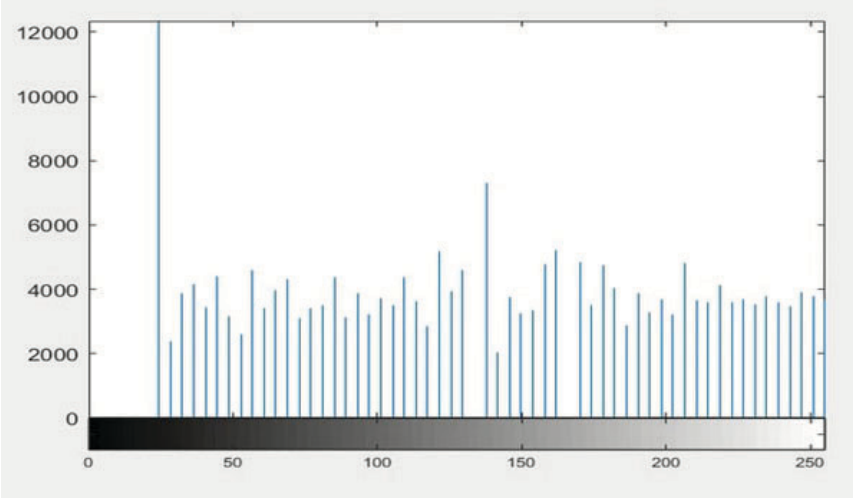

Figure 6: Test Accuracy.

With the help of proposed techniques, we generated a substracted image as shown in Figure 6 and 7 by considering dental images before and after the treatment. ${ }^{19}$

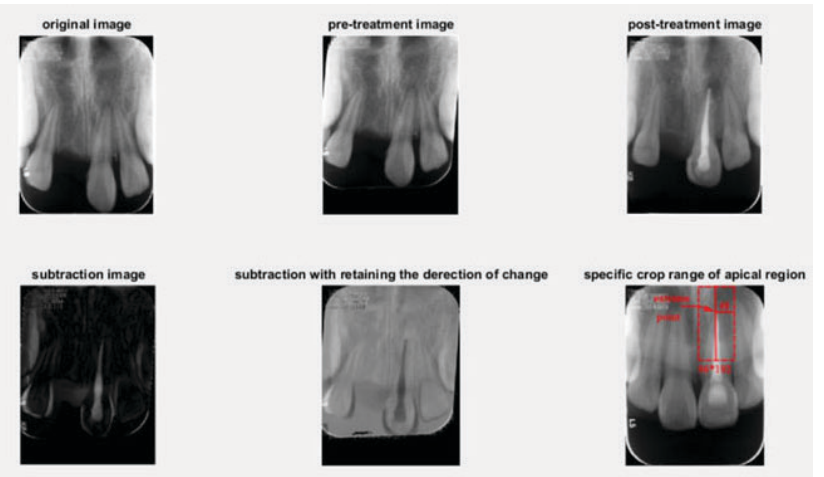

Figure 7: Subtraction image.

The subtracted image is used to find the difference after treating the dental problem. It can be obtained by the difference between the pre-treatment image and post-treatment image. The Apical region ca also be obtained fro this substracted image.

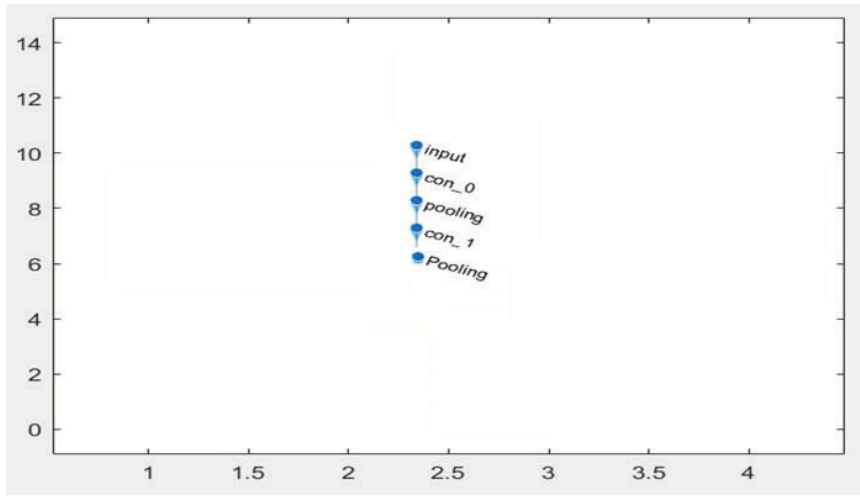

Figure 8: Front Model Prediction.

The architecture of the directed acyclic graph (DAG) network for front model and top model predictions are shown in Figure 8 and Figure 9. These models contain the connected layers of the proposed deep learning technique. The neural network architecture of the proposed system contains layers arranged as a directed acyclic graph.

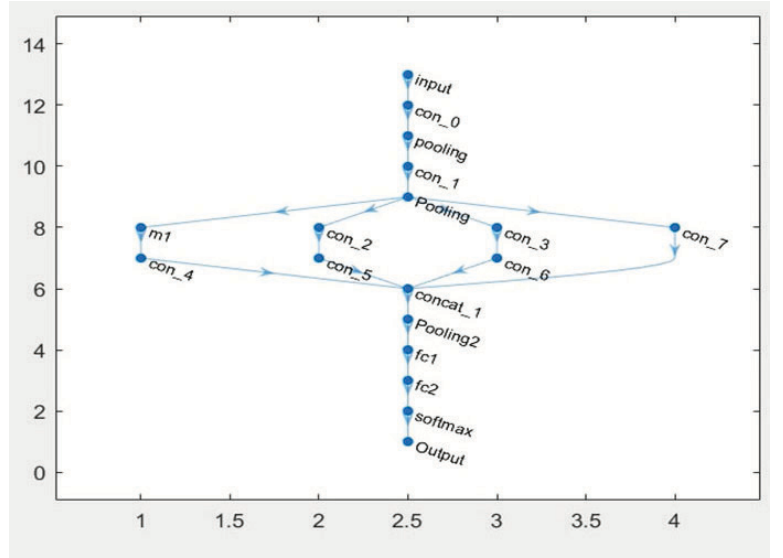

Figure 9: Top Model Prediction. 


\section{DISCUSSION}

From the above results, the dental image analysis approach developed here in this paper incorporates the deep leaning technique for dental image diagnosis. The proposed network architecture with CNN giving more accuracy in dental disease detection. Our research also reports that when planning the data processing measures and interpreting the results of the analysis, analytical skills have to be considered for dental image analysis issues. The proper selection of training and testing data set is to be selected to get high accuracy with the proposed method. we use pictures to incorporate the whole tooth as info. During the assessment, radiologists may make slight alterations depending on the setting. The shooting edge of every tooth is unique, known as the bisecting point procedure. The Scale Invariant Feature Transform (SIFT) and Speed Up Robust Features (SURF) algorithms used in this proposed work identifies the matching points well to obtain the substracted dental image. The dataset of 196 pairs is examined with nearly 12000 iterations to obtain greater accuracy of detecting the dental problems. The proposed machine learning architecture contains connected layers for feature mapping.

\section{CONCLUSION}

As a result of the incredible heterogeneity regarding the picture database sort, results, structures of neural systems, the normalized strategy required to expand the similarity and heartiness between contemplates. We have built up a robotized, smoothed out dental picture investigation approach that incorporates dental picture finding information. It underpins robotized apical area distinguishing proof that spares numerous manual endeavours on information readiness. Our methodology bolsters CNNs for conclusion grouping dependent on little datasets. With constrained marked cases, our methodology yields promising outcomes that are equivalent to master level dental specialists and dental radiologists. Specifically, our technique accomplishes significant outcomes with regards to recognizing 'showing signs of improvement'. Our investigation likewise affirms that for clinical picture examination issues, space information must be viewed as when planning the information explanatory advances and decipher the examination results.

\section{ACKNOWLEDGEMENTS}

Authors acknowledge the immense help received from the scholars whose articles are cited and included in references to this manuscript. The authors are also grateful to authors/ editors/publishers of all those articles, journals and books from where the literature for this article has been reviewed and discussed.
Conflict of interests: We wish to confirm that there are no known conflicts of interest associated with this publication and there has been no significant financial support for this work that could have influenced its outcome.

Funding Sources: None

\section{REFERENCES}

1. Mendonca EA. Clinical decision support systems: Perspectives in dentistry. J. Dent. Educ. 2004;68:589-597.

2. Dmitry V Tuzoff, Lyudmila N Tuzova, Michael M Bornstein, Alexey S Krasnov, Max A Kharchenko, Sergey I Nikolenko, Mikhail M Sveshnikov, Georgiy B Bednenko. Tooth detection and numbering in panoramic radiographs using convolutional neural networks.Dentomaxillofac Radiol.2019;48(4):20180051.

3. Dinggang Shen, Guorong Wu, Heung-ll Suk. Deep Learning in Medical Image Analysis. Annu. Rev. Biomed. Eng. 2017;19:221-248.

4. Hamideh Ehtesham, Reza Safdari, Arash Mansourian, Shahram Tahmasebian, Niloofar Mohammadzadeh, Sara Pourshahidi. Developing a new intelligent system for the diagnosis of oral medicine with case-based reasoning approach. Oral Dis. 2019; 25(6):1555-1563.

5. Topol, E.J. High-performance medicine: the convergence of human and artificial intelligence.Nat. Med.2019;25:44-56.

6. Teruhiko Hiraiwa, Yoshiko Ariji, Motoki Fukuda, Yoshitaka Kise, Kazuhiko Nakata, Akitoshi Katsumata, Hiroshi Fujita, Eiichiro Ariji. A deep-learning artificial intelligence system for assessment of root morphology of the mandibular first molar on panoramic radiography. Dentomaxillofac Radiol. 2019;48(3):20180218.

7. Currie G. Intelligent Imaging: Anatomy of Machine Learning and Deep Learning. J Nucl Med Technol. 2019; 47(4): 273-281.

8. Xue Y, Zhang R, Deng Y, Chen K, Jiang T. A preliminary examination of the diagnostic value of deep learning in hip osteoarthritis. PLoS One. 2017;12(6):e0178992.

9. Judah E S Sklan, Andrew J Plassard, Daniel Fabbri, Bennett A Landman. Toward Content Based Image Retrieval with Deep Convolutional Neural Networks.Proc SPIE Int Soc Opt Eng. 2015;9417:94172C.

10. Falk Schwendickea, Karim Elhennawya, Sebastian Parisa, Philipp Friebertshäusera, Joachim Kroisa. Deep learning for caries lesion detection in near-infrared light transillumination images: A pilot study. J. Dent. 2020;92:103260.

11. Joachim Krois, Thomas Ekert, Leonie Meinhold, Tatiana Golla, Basel Kharbot, Agnes Wittemeier, Christof Dörfer, Falk Schwendicke. Deep Learning for the Radiographic Detection of Periodontal Bone Loss. Sci Rep.2019; 9:8495.

12. Jae-Hong Lee, Do-Hyung Kim, Seong-Nyum Jeong, Seong-Ho Choi. Diagnosis and prediction of periodontally compromised teeth using a deep learning-based convolutional neural network algorithm. J Periodontal Implant Sci.2018; 48(2):114-123.

13. Jae-Hong Lee, Do-Hyung Kim, Seong-Nyum Jeong, SeongHo Choi. Detection and diagnosis of dental caries using a deep learning-based convolutional neural network algorithm. J Dent. 2018; 77:106-111.

14. Falk Schwendicke, Tatiana Golla, Martin Dreher, Joachim Krois. Convolutional neural networks for dental image diagnostics: A scoping review. J Dent.2019; 91:103226.

15. Hu Chen, Kailai Zhang, Peijun Lyu, Hong Li, Ludan Zhang, Ji $\mathrm{Wu}, \mathrm{Chin}-\mathrm{Hui}$ Lee. A deep learning approach to automatic teeth 
detection and numbering based on object detection in dental periapical films. Sci Rep. 2019; 9(1):3840.

16. Mahoor MH, Abdel-Mottaleb M. Classification and numbering of teeth in dental bitewing images. Pattern Recognition. 2005; 38(4):577-586.

17. Thomas Ekert, Joachim Krois, Leonie Meinhold, Karim Elhennawy, Ramy Emara, Tatiana Golla, Falk Schwendicke. Deep Learning for the Radiographic Detection of Apical Lesions. J Endod. 2019; 45(7):917-922.e5.
18. Cosimo Nardi, Linda Calistri, Giulia Grazzini, Isacco Desideri, Chiara Lorini, Mariaelena Occhipinti, Francesco Mungai, Stefano Colagrande. Is Panoramic Radiography an Accurate Imaging Technique for the Detection of Endodontically Treated Asymptomatic Apical Periodontitis?. J Endod. 2018; 44(10):15001508 .

19. Kailai Zhang, Ji Wu, Hu Chen, Peijun Lyu. An effective teeth recognition method using label tree with cascade network structure. Comput Med Imaging Graph.2018;68:61-70. 